\title{
УДК 621.3.011.71
}

\section{С.А. Курганов, И.А. Прокофьев}

\section{ФОРМУЛА ДЛЯ НАХОЖДЕНИЯ ФУНКЦИЙ ЧУВСТВИТЕЛЬНОСТИ ЛИНЕЙНЫХ ЭЛЕКТРИЧЕСКИХ ЦЕПЕЙ ПО ЧАСТЯМ}

Курганов Сергей Александрович, доктор технических наук, окончил Ульяновский политехнический институт по специальности "Конструирование и производство радиоаппаратуры», профрессор кафедры «Электроснабжение» Ульяновского государственного технического университета. Имеет статьи и монографии в области теоретических основ электротехники. [e-mail: sak@ulstu.ru].

Прокофьев Иван Алексеевич, окончил бакалавриат по направлению «Электроэнергетика и электротехника» УлГТУ, магистрант кафедры «Электроснабжение» УлГТУ. Имеет статьи в области теоретических основ электротехники. [e-mail: prokofev.ivan.2015@yandex.ru].

\section{Аннотация}

Предложена формула для нахождения символьных выражений чувствительности произвольных линейных электрических цепей по частям - делением схемы на подсхемы. Электрические цепи могут содержать как двухполюсные элементы, так и управляемые источники (УИ) напряжения и тока, в том числе идеальные УИ - нуллоры. Деление схемы выполняется на основе эквивалентного многомерного генератора (ЭМГ) методом схемно-алгебраической редукции. Чувствительность схемы находится с использованием частных чувствительностей элементов ЭМГ или их числителей, которые формируются с помощью разностной схемно-алгебраической формулы чувствительности. При этом исключены сокращающиеся и нулевые схемные миноры. Формула требует для нахождения символьных функций относительной чувствительности раскрытия многократно меньшего числа определителей подсхем - схемных миноров, чем известная формула чувствительности на основе бисекции определителей. Приведен пример нахождения функций чувствительности двухкаскадного активного фильтра.

Ключевые слова: электрические цепи, чувствительность, символьная функция, анализ по частям, схемный определитель, частная чувствительность, эквивалентный многомерный генератор, схемный минор.

doi:

\section{FORMULA FOR FINDING THE SENSITIVITY FUNCTIONS OF LINEAR ELECTRICAL CIRCUITS IN PARTS}

Sergei Aleksandrovich Kurganov, Doctor of Sciences in Engineering; graduated from Ulyanovsk Polytechnic Institute with a degree in Design and Production of Radio Equipment; Professor of the Department of Electric Supply of Ulyanovsk State Technical University; an author of articles and monographs in the field of theoretical foundations of electrical engineering. e-mail: sak@ulstu.ru.

Ivan Alekseevich Prokofev, graduated from Ulyanovsk State Technical University with Bachelor's degree in Electric Power and Electrical Engineering; Master Student of the Department of Electric Supply of UISTU; an author of articles in the field of theoretical foundations of electrical engineering. e-mail: prokofev.ivan.2015@yandex.ru.

\begin{abstract}
A formula for finding symbolic expressions of sensitivity of arbitrary linear electrical circuits in parts by dividing the circuit into subsystems is proposed. Electrical circuits can contain both bipolar elements and controlled sources (UIs) of voltage and current including ideal Uls - nullors. The scheme division is performed on the basis of an equivalent multidimensional generator (EMG) by the scheme-algebraic reduction method. The circuit sensitivity formula contains the sensitivity quotients of the EMG elements or their numerators, which are found using the difference circuit-algebraic sensitivity formula. The proposed formula excludes reducing multipliers and zero terms, and it requires to find the symbolic functions of relative sensitivity of the disclosure of a much smaller number of determinants of subcircuits-circuit minors than the known sensitivity formula based on bisection of determinants.
\end{abstract}

Key words: electrical circuits, sensitivity, symbolic function, piecemeal analysis, circuit determinant, partial sensitivity, equivalent multidimensional generator, circuit minor. 


\section{Введение}

Относительная чувствительность схемной функции $F$ по параметру $w$ определяется выражением [1]:

$S_{w}^{F}=\frac{d F}{d w} \frac{w}{F}$.

Символьные функции, формируемые непосредственно по формуле (1) на основе: узловых уравнений в расширенном координатном базисе [2], графовых моделей [3] и матрицы трансимпедансов [4], содержат избыточные выражения - одинаковые слагаемые с противоположными знаками - из-за наличия повторяющихся параметров в матричных и графовых моделях. При явном выделении варьируемого параметра $w$ из числителя и знаменателя схемной функции $F$ получается разностная формула чувствительности, содержащая разность произведений частей числителя и знаменателя схемной функции [5], а при использовании тождества Якоби - обобщенная формула Боде в виде отношения двух попарных произведений схемных определителей или многократных суммарных алгебраических дополнений (МСАД) [6].

При раскрытии МСАД в обобщенной формуле Боде методом схемных определителей (МСО) формируются безызбыточные символьные выражения чувствительности [6]. Безызбыточные выражения получаются и по разностной формуле, если использовать операции выделения параметров из разности определителей [7]. При этом разностная формула требует раскрытия всего двух определителей - числителя и знаменателя схемной функции - вместо четырех по обобщенной формуле Боде.

О нахождении чувствительности делением схемы на части. Численный расчет чувствительности по частям (диакоптика) предложен на основе эквивалентного многомерного генератора (ЭМГ), параметры которого находятся путем матричного обращения узловой или гибридной матрицы в [8]. Однако применение матричного подхода для символьного анализа чувствительности приводит, как отмечалось выше, к избыточным выражениям.

Безызбыточный символьный анализ чувствительности по частям основан на бисекции схемных определителей в обобщенной формуле Боде, при этом определители находятся через схемные миноры - определители подсхем [9]. Экономия вычислительных операций достигается не только за счет нахождения определителей по частям, но и за счет сокращения одинаковых схемных миноров в числителе и знаменателе формулы чувствительности, и исключения повторного раскрытия одинаковых миноров. Недостатком подхода является резкий рост числа миноров при возрастании числа полюсов у подсхем.

Многократно меньше схемных миноров требуется для нахождения параметров ЭМГ, которые используются при символьном анализе электрических цепей методом схемно-алгебраической редукции (САР) [10]. Желательно использовать САР и для символьного анализа чувствительности.

Целью статьи является снижение числа вычислительных операций при символьном анализе чувствительности по частям за счет уменьшения числа раскрываемых схемных миноров.

\section{1 ФОРМУЛА ДЛЯ ОТНОСИТЕЛЬНОЙ ЧУВСТВИТЕЛЬНОСТИ НА} ОСНОВЕ ЧАСТНЫХ ЧУВСТВИТЕЛЬНОСТЕЙ ПАРАМЕТРОВ ЭМГ

Исходную схему с источником электродвижущей силы (ЭДС) $\underline{E}$ (рис. 1a) разделим на две подсхемы (рис. 1б) так, чтобы элемент с варьируемым параметром - источник тока, управляемый напряжением (ИТУН), оказался в подсхеме 1 с входным источником. Эту подсхему представим в виде ЭМг с $Z$-параметрами (рис. 2).

Коэффициент влияния (абсолютная чувствительность) параметра $Y$ на передаточную функцию напряжения $\underline{K}=\underline{U}_{\text {вых }} / \underline{E}$ схемы на рисунке 2 определяется через коэффициенты влияния параметров ЭМГ по формуле [8]:

$$
\begin{aligned}
A_{Y}^{K} & =\frac{d K}{d Y}= \\
& =\sum_{i=1}^{n} \sum_{j=1}^{n} A_{Z_{i j}}^{K} A_{Y}^{Z_{i j}}+\sum_{i=1}^{n} A_{E_{i}}^{K} A_{Y}^{E_{i}},
\end{aligned}
$$

где $A_{Z_{i j}^{\prime}}^{K} A_{E_{i}}^{K}$ - коэффициенты влияния сопротивления $Z_{i j}$ и ЭДС $E_{i}$ на функцию $K$;

$A_{Y}^{Z_{i j}}, A_{Y}^{E_{i}}$ - коэффициенты влияния проводимости ИтУн $Y$ на функции сопротивления $Z_{i j}$ и ЭДс $E_{i}$;

$n$ - число сторон подсхемы 1 на рисунке 2.

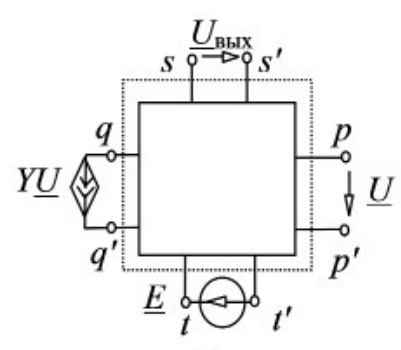

a

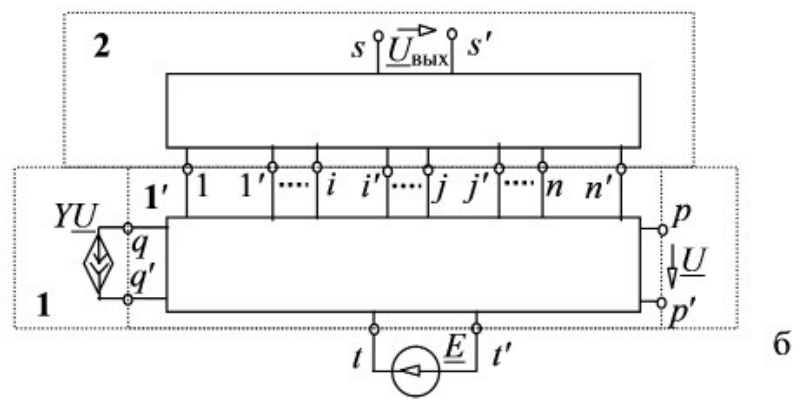

Рис. 1. Электрическая цепь для нахождения чувствительности по параметру ИтУН: исходная (а) и разделенная на подсхемы 1 и 2 со сторонами $1-1^{\prime}, \ldots, i-i^{\prime}, \ldots, j-j^{\prime}, \ldots, n-n^{\prime}$ (б) 


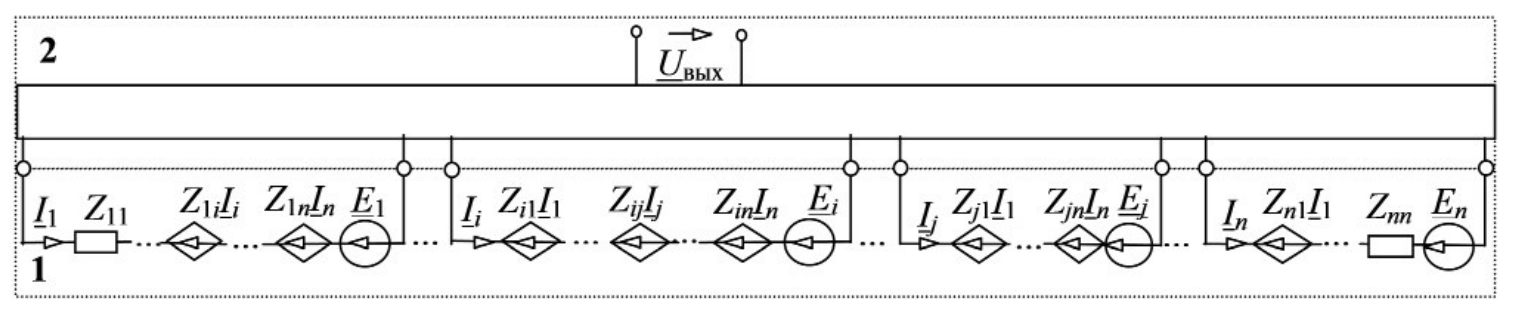

Рис. 2. Схема замещения цепи на рисунке 16 с подсхемой 1 в виде ЭМГ с $Z$-параметрами: $Z_{i j}$ - параметр источника напряжения, управляемого током (ИНУТ), при $i \neq j$

Из формулы (2) можно получить формулу для нахождения функции относительной чувствительности коэффициента $K$ через частные относительные чувствительности ЭМГ путем формальной замены символа $A$ коэффициента влияния на символ $S$ относительной чувствительности:

$$
S_{Y}^{K}=\sum_{i=1}^{n} \sum_{j=1}^{n} S_{Z_{i j}}^{K} S_{Y}^{Z_{i j}}+\sum_{i=1}^{n} S_{E_{i}}^{K} S_{Y}^{E_{i}}
$$

где $S_{Z_{i j^{\prime}}}^{K} S_{E_{i^{\prime}}}^{K} S_{Y}^{Z_{i j}}, S_{Y}^{E_{i}}$ - обозначения частных относительных чувствительностей, в которых функция и варьируемый параметр, как и в коэффициентах влияния из формулы (2), указаны в верхнем и нижнем индексе.
Для символьного раскрытия частных чувствительностей в (3) более предпочтительна разностная формула чувствительности [7], она требует, как уже отмечалось выше, раскрытия всего двух определителей вместо четырех по обобщенной формуле Боде.

\section{2 БЕЗЫЗБЫТОЧНАЯ РАЗНОСТНАЯ ФОРМУЛА чУВСТВИТЕЛЬНОсти [7]}

Для схемы на рисунке 1а разностная схемно-алгебраическая формула (САФ) чувствительности передаточной функции напряжения $K=\underline{U}_{\text {вых }} / \underline{E}$ по параметру ИтУн $Y$ имеет вид:

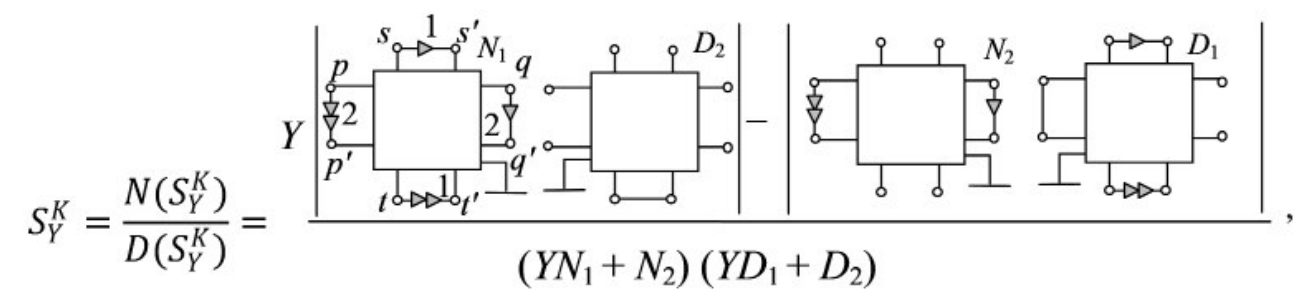

где $N\left(S_{Y}^{K}\right)$ и $D\left(S_{Y}^{K}\right)$ - определители числителя и знаменателя функции чувствительности; пара вертикальных линий обозначает определитель соответствующей схемы; каждое произведение из двух схемных определителей объединено в один определитель; одинарной и двойной заштрихованной стрелкой показан нуллатор и норатор соответственно; цифрой 1 (2) пронумерованы одноименные норатор и нуллатор; $N_{1}, N_{2}$ и $D_{1}, D_{2}$ части числителя $N(K)$ и знаменателя $D(K)$ функции $K$, следующие из формул выделения параметра $Y$ :

$$
N(K)=Y N_{1}+N_{2} \text { и } D(K)=Y D_{1}+D_{2} .
$$

Схема числителя $N(K)$ (знаменателя $D(K))$ получается путем подключения норатора и нуллатора к входу и выходу (к входу) исходной схемы на рисунке 1а. Cхемы $N_{1}$ и $N_{2}\left(D_{1}\right.$ и $\left.D_{2}\right)$ формируются из схемы числителя (знаменателя) путем замены ИТУН нуллором и удаления его из схемы.

Формула (4) может быть записана компактно через МСАД:

$$
S_{Y}^{K}=Y \frac{\Delta_{\left(t+t^{\prime}\right)\left(s+s^{\prime}\right),\left(q+q^{\prime}\right)\left(p+p^{\prime}\right)} \Delta_{\left(t+t^{\prime}\right)\left(t+t^{\prime}\right)}-\Delta_{\left(t+t^{\prime}\right)\left(s+s^{\prime}\right)} \Delta_{\left(t+t^{\prime}\right)\left(t+t^{\prime}\right),\left(q+q^{\prime}\right)\left(p+p^{\prime}\right)}}{\left(Y \Delta_{\left(t+t^{\prime}\right)\left(s+s^{\prime}\right),\left(q+q^{\prime}\right)\left(p+p^{\prime}\right)}+\Delta_{\left(t+t^{\prime}\right)\left(s+s^{\prime}\right)}\right)\left(Y \Delta_{\left(t+t^{\prime}\right)\left(t+t^{\prime}\right),\left(q+q^{\prime}\right)\left(p+p^{\prime}\right)}+\Delta_{\left(t+t^{\prime}\right)\left(t+t^{\prime}\right)}\right)},
$$

где $\Delta$ - определитель схемы на рисунке 1а, ограниченной пунктиром; $\Delta_{\left(t+t^{\prime}\right)\left(s+s^{\prime}\right),\left(q+q^{\prime}\right)\left(p+p^{\prime}\right)}$ - МСАД, у которого первая и вторая пара символов в каждой паре скобок означает полюса подключения норатора и нуллатора в соответствующей схеме (4).
Символьные выражения чувствительности формируются по разностной формуле (4) путем рекурсивного выделения параметров из схемных определителей $N_{1}$, $N_{2}$ и $D_{1}, D_{2}$. Для исключения избыточных одинаковых слагаемых с противоположными знаками, которые 
имеют место в числителе (6), одинаковые параметры выделяются одновременно из разности в числителе. При этом избыточные слагаемые локализуются в паре одинаковых схемных миноров с противоположными знаками, которые удаляются из САФ [7].

\section{3 САР ДЛЯ НАХОЖДЕНИЯ СИМВОЛЬНЫХ ПАРАМЕТРОВ} ЭМГ В ФОРМУЛЕ (3) ЧУВСТВИТЕЛЬНОСТИ ПО ЧАСТЯМ

САР называется операция замены подсхемы ЭМГ, параметры которого находятся в символьном виде на основе схемных определителей [10]. Для подсхемы 1 на рисунке 16 параметры ЭМГ - передаточное сопротивление $Z_{i j}$ и ЭДс $\underline{E}_{i}$ (рис. 2) - получаются по следующим МСАД-формулам:

$$
\begin{aligned}
& Z_{i j}=\frac{d_{\left(t+t^{\prime}\right)\left(t+t^{\prime}\right),\left(j+j^{\prime}\right)\left(i+i^{\prime}\right)}}{d_{\left(t+t^{\prime}\right)\left(t+t^{\prime}\right)}} \\
& \underline{E}_{i}=\kappa_{i} E ; \kappa_{i}=\frac{d_{\left(t+t^{\prime}\right)\left(i+i^{\prime}\right)}}{d_{\left(t+t^{\prime}\right)\left(t+t^{\prime}\right)}}
\end{aligned}
$$

где $d$ - определитель подсхемы 1 на рисунке $16 ; \kappa_{i}-$ коэффициент передачи напряжения от источника $\underline{E}$ к стороне $i$ подсхемы $\mathbf{1}$.

Формула (3) для чувствительности по частям обладает избыточностью - наличием нулевых и повторяющихся определителей из-за пар частных чувствительностей $S_{Y}^{Z_{i j}}$ и $S_{Y}^{E_{i}}\left(S_{E_{i}}^{K}\right.$ и $\left.S_{Z_{i j}}^{K}\right)$, каждая из которых находится по одной и той же подсхеме 1 на рисунке 16 (схеме с ЭМг на рисунке 2).

\section{4 ВЫВОД БЕЗЫЗБЫТОЧНОЙ ФОРМУЛЫ ДЛЯ НАХОЖДЕНИЯ} СИМВОЛЬНОЙ ЧУВСТВИТЕЛЬНОСТИ СХЕМЫ НА ОСНОВЕ ЧАСТНЫХ ЧУВСТВИТЕЛЬНОСТЕЙ ПАРАМЕТРОВ ЭМГ

Формулы частных чувствительностей $S_{E_{i}}^{K}$ и $S_{Z_{i j}}^{K}$ для чувствительности $S_{Y}^{K}$ из (3) находятся по схеме на рисунке 3 , которая получается на основе неявного принципа наложения [11] путем замены независимых источников ЭДС $\underline{E}_{1}, \underline{E}_{2}, \ldots, \underline{E}_{v}$ на рисунке 2 источниками напряжения, управляемыми напряжением (ИНУН) разомкнутого источника ЭДС $\underline{E}$, подключенного к произвольному узлу цепи. Параметры ИНУН $\kappa_{1}, \kappa_{2}, \ldots, \kappa_{n}$ находятся по формуле (9) как коэффициенты передачи напряжения подсхемы 1' (рис. 1б) от источника ЭдС к соответствущей стороне.
Для схемы на рисунке 3 коэффициент передачи напряжения записывается по аналогии с формулой (9):

$$
K=\frac{\underline{U}_{\text {вых }}}{\underline{E}}=\frac{N(K)}{D(K)}=\frac{\delta_{\left(t+t^{\prime}\right)\left(s+s^{\prime}\right)}}{\delta_{\left(t+t^{\prime}\right)\left(t+t^{\prime}\right)}},
$$

где $\delta$ - определитель схемы на рисунке 3.

Из числителя $N(K)$ и знаменателя $D(K)$ в (10) получаются подобно (6) части числителя и знаменателя для чувствительности $S_{Z_{i j}}^{K}$ :

$$
\begin{aligned}
& N_{1}\left(K, Z_{i j}\right)=\delta_{\left(t+t^{\prime}\right)\left(s+s^{\prime}\right),\left(p+p^{\prime}\right)\left(q+q^{\prime}\right)} \\
& N_{2}\left(K, Z_{i j}\right)=\delta_{\left(t+t^{\prime}\right)\left(s+s^{\prime}\right)}^{Z_{i j}=0} \\
& D_{1}\left(K, Z_{i j}\right)=\delta_{\left(t+t^{\prime}\right)\left(t+t^{\prime}\right),\left(p+p^{\prime}\right)\left(q+q^{\prime}\right)} \\
& D_{2}\left(K, Z_{i j}\right)=\delta_{\left(t+t^{\prime}\right)\left(t+t^{\prime}\right)}^{Z_{i j}=0}
\end{aligned}
$$

где первая и вторая переменная в скобках при символах $N_{1}, N_{2}, D_{1}$ и $D_{2}$ показывает их принадлежность к схемной функции и выделенному по формуле (5) параметру; индекс $Z_{i j}=0$ означает, что МСАД находится при нулевом параметре ИНУТ.

чувствительность $S_{E_{i}}^{K}$ совпадает с чувствительностью $S_{\kappa_{i}}^{K}$, поскольку функция $K$ не зависит от входной эдс $\underline{E}$. Необходимые для нахождения $S_{\kappa_{i}}^{K}$ части $N_{1}\left(K, \kappa_{i}\right)$, $N_{2}\left(K, \kappa_{i}\right)$ и $D_{1}\left(K, \kappa_{i}\right), D_{2}\left(K, \kappa_{i}\right)$ следуют, как и для $S_{Z_{i j}}^{K}$ из числителя и знаменателя функции $K$ в (10). Знаменатель $D(K)$ в (10) не содержит ИНУН с параметром $\kappa_{i}$, поэтому $D_{1}\left(K, \kappa_{i}\right)=0$, а $D_{2}\left(K, \kappa_{i}\right)=D(K)$. в результате в числителе и знаменателе разностной формулы (4) применительно к чувствительности $S_{E_{i}}^{K}$ сокращается определитель $D(K)$, и формула принимает вид:

$$
\begin{aligned}
S_{E_{i}}^{K}=S_{\kappa_{i}}^{K} & =\kappa_{i} \frac{N_{1}\left(K, \kappa_{i}\right)}{N(K)}= \\
& =\kappa_{i} \frac{N_{1}\left(K, \kappa_{i}\right)}{\kappa_{i} N_{1}\left(K, \kappa_{i}\right)+N_{2}\left(K, \kappa_{i}\right)} .
\end{aligned}
$$

Части числителя $K$ в (12) находятся аналогично (11) и имеют вид:

$$
\begin{aligned}
& N_{1}\left(K, \kappa_{i}\right)=\delta_{\left(t+t^{\prime}\right)\left(s+s^{\prime}\right),\left(r+r^{\prime}\right)\left(t+t^{\prime}\right)} \\
& N_{2}\left(K, \kappa_{i}\right)=\delta_{\left(t+t^{\prime}\right)\left(s+s^{\prime}\right)}^{\kappa_{i}=0}
\end{aligned}
$$

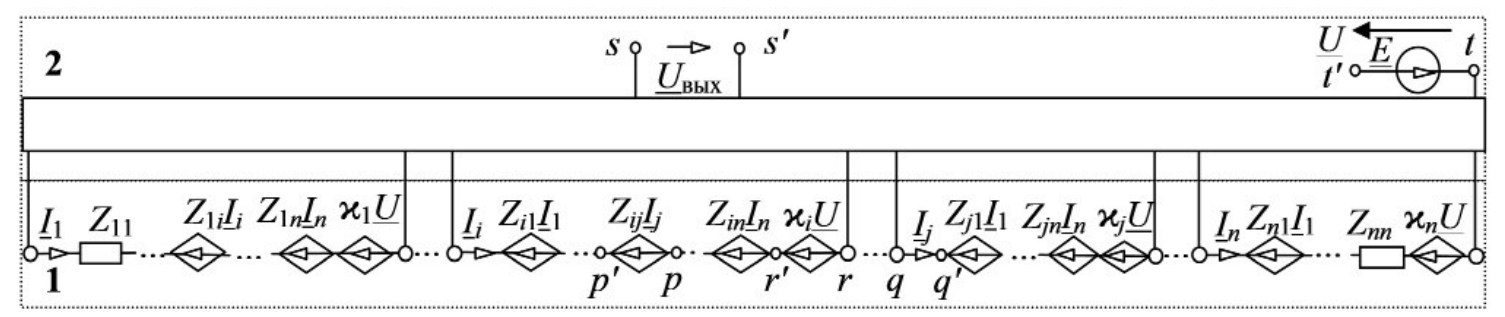

Рис. 3. Схема цепи на рисунке 2 после замены независимых источников ЭМг УИ напряжения 
Определитель $N(K)$ в (12) не зависит от выделяемого параметра $\kappa_{i}$, поэтому для его нахождения используется пара $N_{1}\left(K, \kappa_{i}\right)$ и $N_{2}\left(K, \kappa_{i}\right)$ для произвольного $i$.

Формулы для частных чувствительностей $S_{Y}^{Z_{i j}}$, $\boldsymbol{S}_{\boldsymbol{Y}}^{\boldsymbol{E}_{\boldsymbol{i}}}$. Формулы $N_{1}, N_{2}, D_{1}$ и $D_{2}$ для получения $S_{Y}^{Z_{i j}}$ и $S_{Y}^{E_{i}}$ по разностной формуле (4) находятся аналогично формулам (11) и (13) путем выделения и нейтрализации варьируемого параметра $Y$ в МсАД-формулах числителя и знаменателя передаточного сопротивления $Z_{i j}$ (7) и коэффициента $\kappa_{i}$ (9) и имеют вид:

$N_{1}\left(Z_{i j}, Y\right)=\sigma_{\left(t+t^{\prime}\right)\left(t+t^{\prime}\right),\left(j+j^{\prime}\right)\left(i+i^{\prime}\right),\left(q+q^{\prime}\right)\left(p+p^{\prime}\right)}$;

$N_{2}\left(Z_{i j}, Y\right)=\sigma_{\left(t+t^{\prime}\right)\left(t+t^{\prime}\right),\left(j+j^{\prime}\right)\left(i+i^{\prime}\right)}$;

$N_{1}\left(\kappa_{i}, Y\right)=\sigma_{\left(t+t^{\prime}\right)\left(i+i^{\prime}\right),\left(q+q^{\prime}\right)\left(p+p^{\prime}\right)}$;

$N_{2}\left(\kappa_{i}, Y\right)=\sigma_{\left(t+t^{\prime}\right)\left(i+i^{\prime}\right)}$;

$D_{1}\left(Z_{i j}, Y\right)=D_{1}\left(\kappa_{i}, Y\right)=$

$$
\begin{gathered}
=\sigma_{\left(t+t^{\prime}\right)\left(t+t^{\prime}\right),\left(q+q^{\prime}\right)\left(p+p^{\prime}\right)} ; \\
D_{2}\left(Z_{i j}, Y\right)=D_{2}\left(\kappa_{i}, Y\right)=\sigma_{\left(t+t^{\prime}\right)\left(t+t^{\prime}\right)} \\
D\left(Z_{i j}, Y\right)=D\left(\kappa_{i}, Y\right)= \\
=Y D_{1}\left(Z_{i j}, Y\right)+D_{2}\left(Z_{i j}, Y\right)=d,
\end{gathered}
$$

где $\sigma$ - определитель подсхемы $\mathbf{1}^{\prime}$ на рисунке $16 ; d$ как и ранее, определитель подсхемы 1 на рисунке 16.

После подстановки формул (11)-(12) и (14) в (3) вынесем варьируемый параметр и определитель $d$ за знак суммы и получим искомую формулу относительной чувствительности без сокращающихся сомножителей и нулевых слагаемых:

$$
\begin{aligned}
S_{Y}^{K}=\frac{Y}{d}\left(\sum_{i=1}^{n} \sum_{j=1}^{n} \frac{S_{Z_{i j}}^{K} N\left(S_{Y}^{Z_{i j}}\right)}{N\left(Z_{i j}\right)}+\right. \\
\left.+\sum_{i=1}^{n} \frac{\kappa_{i} N_{1}\left(K, \kappa_{i}\right) N\left(S_{Y}^{\kappa_{i}}\right)}{N(K) N\left(\kappa_{i}\right)}\right) .
\end{aligned}
$$

Если схема делится по двум узлам $(n=1)$, то формула (15) упрощается: $N_{1}\left(K, Z_{11}\right)=0$ из-за последовательного соединения норатора и сопротивления $Z_{11}$ в схеме числителя функции К в формуле (10), а $N_{2}\left(K, Z_{11}\right)$ сокращается в числителе и знаменателе $S_{Z_{i j}}^{K}$. Также $N_{2}\left(K, \kappa_{1}\right)=0$, поскольку при нейтрализации ИНУН с параметром $\kappa_{i}$ норатор (в схеме числителя вместо входного источника) оказывается разомкнутым. После удаления нулевых слагаемых и сокращения одинаковых сомножителей в числителе и знаменателе формула (15) приводится к виду:

$$
S_{Y}^{K}=\frac{Y}{d}\left(-\frac{D_{1}\left(K, Z_{11}\right) N\left(S_{Y}^{\kappa_{1}}\right)}{d D(K)}+\frac{N\left(S_{Y}^{\kappa_{1}}\right)}{N\left(\kappa_{1}\right)}\right) .
$$

\section{5 СРАВНЕНИЕ ЧИСЛА МИНОРОВ В ОБОБЩЕННОЙ ФОРМУЛЕ}

\section{БОДЕ [9] И ФОРМУЛЕ НА ОСНОВЕ ЭМГ}

При использовании бисекции в обобщенной формуле Боде получается 8 подсхем, из которых 6 подсхем разных [9]. Каждая подсхема характеризуется числом $\mu$ миноров (строка 2 в таблице) [9], поэтому общее число миноров $b=6 \mu$ (строка 3 в таблице).

В предлагаемой формуле (15) с частными чувствительностями параметров ЭМГ число миноров $\gamma$ включает: $n^{2}$ ( $n=v-1$ число независимых полюсов или сторон подсхемы) схемных миноров числителей для сопротивлений $Z_{i j}(i, j=1 . . n) ; n$ миноров числителей для коэффициентов $\kappa_{i}$ и минор их общего знаменателя; $n^{2}+n$ миноров числителя $N(K)$ и знаменателя $D(K)$ исследуемой схемной функции с разложением по каждому $Z_{i j}$ и $\kappa_{i}$. В результате $\gamma=2\left(n^{2}+n\right)+1$ (строка 4 в таблице). При этом для $v=2$ число $\gamma=4$, поскольку схемный минор $N(K)$ в формуле чувствительности (16) отсутствует.

Как видно из строк 3 и 4 таблицы, число раскрываемых схемных миноров по формуле (15) многократно меньше числа схемных миноров по бисекционной формуле [9].

Таблица

Сравнение числа миноров в бисекционной формуле чувствительности

и предлагаемой формуле на основе частных чувствительностей ЭМГ

\begin{tabular}{|l|c|c|c|c|c|c|c|c|c|}
\hline Число полюсов, $v$ & 2 & 3 & 4 & 5 & 6 & 7 & 8 & 9 & 10 \\
\hline Число миноров подсхемы, $\mu$ & 2 & 6 & 20 & 70 & 252 & 924 & 3432 & 12870 & 48620 \\
\hline $\begin{array}{l}\text { Число миноров в бисекционной } \\
\text { формуле чувствительности [9], } b\end{array}$ & 12 & 36 & 120 & 420 & 1512 & 5544 & 20592 & 77220 & 291720 \\
\hline $\begin{array}{l}\text { Число миноров в формуле (15) } \\
\text { на основе ЭМГ, } \gamma\end{array}$ & 4 & 13 & 15 & 23 & 33 & 45 & 59 & 75 & 93 \\
\hline
\end{tabular}




\section{6 ПРИМЕР ФОРМИРОВАНИЯ ФУНКЦИИ ЧУВСТВИТЕЛЬНОСТИ ФИЛЬТРА НИЖНИХ ЧАСТОТ}

Схема фильтра четвертого порядка представлена на рисунке 4 [2]. Операционные усилители (ОУ) $k_{3}$ и $k_{4}$ моделируются с помощью ИНУН с одноименными действительными коэффициентами передачи напряжения и выходными сопротивлениями $R_{3}$ и $R_{4}$. Требуется найти символьное выражение чувствительности $S_{C_{5}}^{K}$ коэффициента передачи напряжения

$K=U_{\text {вых }} / U_{\text {вх }}$ при варьировании емкости $C_{5}$.

Схема на рисунке 4 делится по двум узлам 9 и 0 на две подсхемы с числом сторон $n=1$, поэтому чувствительность определяется по формуле (16) при $Y=y_{5}$.

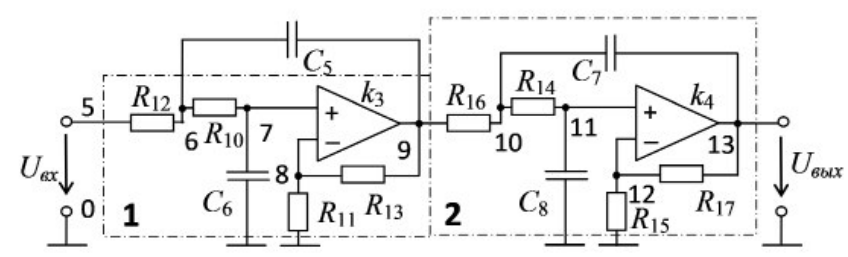

Рис. 4. Активный фильтр нижних частот четвертого порядка: 1, 2 - номера подсхем

Выражение $D_{1}\left(K, Z_{11}\right)$ для формулы (16) находится из схемы на рисунке 5а при $\underline{U}_{\mathrm{BX}}=0$ и удаленном сопротивлении $Z_{11}$ :

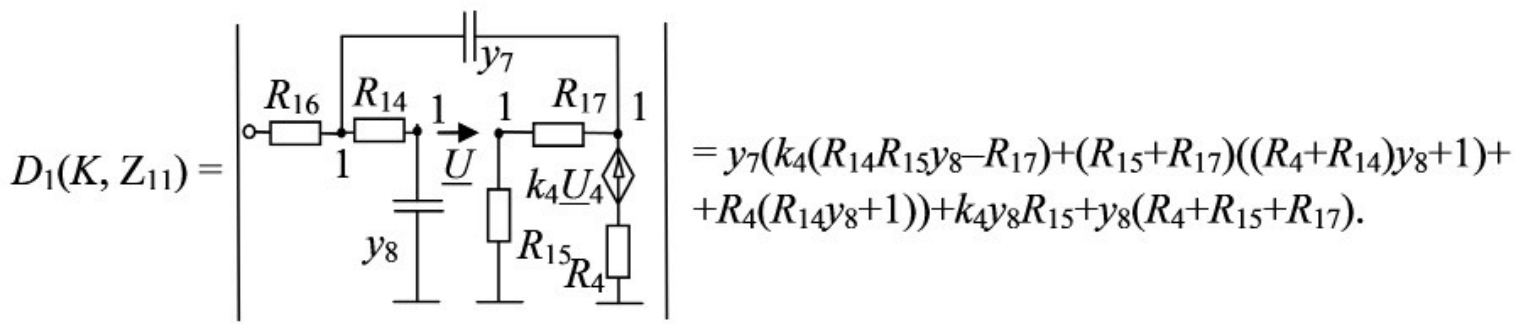

Символьная формула (17) получена по МСО путем последовательного выполнения операций: выделения проводимости $y_{7}$, параметра ИНУН $k_{4}$ и проводимости $y_{8}$; сложения последовательно соединенных сопротивлений $R_{4}, R_{15}$ и $R_{17}, R_{15}$ и $R_{17}, R_{4}$ и $R_{14}$; выделения последовательно соединенных сопротивления $R_{14}$ и проводимости $y_{8}$, сопротивлений $R_{4}, R_{14}$ и проводимости $y_{8}$.

САФ $D_{2}\left(K, Z_{11}\right)$ получается из той же схемы на рисунке 5а, как и $D_{1}\left(K, Z_{11}\right)$, путем нейтрализации $Z_{11}$.
При разложении определителя используется выражение $D_{1}\left(K, Z_{11}\right)$ из (17), в результате

$$
\begin{aligned}
& D_{2}\left(K, Z_{11}\right)=R_{16} D_{1}(K)+\left(R_{14} y_{8}+1\right) \times \\
& \quad \times\left(k_{4} R_{15}+\left(R_{15}+R_{17}\right)\left(R_{14} y_{7}+1\right)+R_{4}\right) .
\end{aligned}
$$

Определитель исследуемой схемы с эквивалентным генератором (рис. 5а) находится по формуле вида (5):

$$
D=Z_{11} D_{1}\left(K, Z_{11}\right)+D_{2}\left(K, Z_{11}\right) .
$$

Сопротивление $Z_{11}$ из (19) формируется при нахождении частной чувствительности $S_{y_{5}}^{Z_{11}}$. В соответствии с (7) и (9) МСАД числителей функций $S_{y_{5}}^{Z_{11}}$ и $S_{y_{5}}^{E_{11}}$ имеют вид:

$$
\begin{aligned}
& N\left(S_{y_{5}}^{Z_{11}}\right)=y_{5}\left(\delta_{(5+0)(5+0),(9+0)(9+0),(6+9)(6+9)} \delta_{(5+0)(5+0)}-\delta_{(5+0)(5+0),(9+0)(9+0)} \delta_{(5+0)(5+0),(6+9)(6+9)}\right) \\
& N\left(S_{y_{5}}^{E_{11}}\right)=N\left(S_{y_{5}}^{\mathrm{K}_{1}}\right)=y_{5}\left(\delta_{(5+0)(9+0),(6+9)(6+9)} \delta_{(5+0)(5+0)}-\delta_{(5+0)(9+0)} \delta_{(5+0)(5+0),(6+9)(6+9)}\right)
\end{aligned}
$$

где $\delta$ - определитель одноименной подсхемы на рисунке 56.
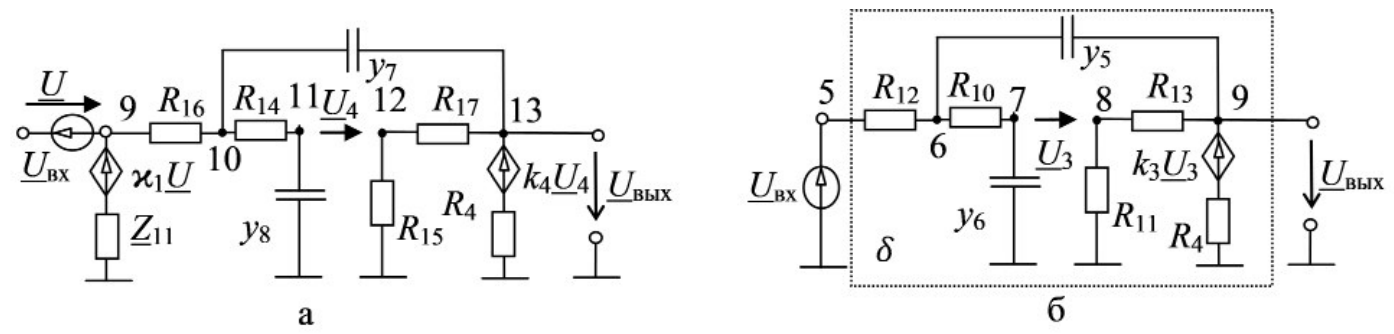

Рис. 5. Исходная цепь (рис. 4) с подсхемой 1 в виде ЭМг в $Z$-параметрах (а) и подсхема 1 (б) 
Применяя к (20) и (21) одновременное выделение одинаковых параметров из разности [7], получаем безызбыточные выражения:

$$
\begin{aligned}
& N\left(S_{y_{5}}^{Z_{11}}\right)=\left(R_{11}+R_{13}\right)^{2}\left(\left(R_{12}+R_{10}\right) y_{6}+1\right) R_{3}\left(-R_{3}\left(R_{12}+R_{10}\right) y_{6}+k_{3} R_{12}-R_{3}\right) \\
& N\left(S_{y_{5}}^{E_{11}}\right)=-\left(R_{11}+R_{13}\right)\left(-\left(\left(R_{10}+R_{12}\right) y_{6}+1\right) R_{3}+k_{3} R_{12}\right) \times \\
& \quad \times\left(\left(R_{10} y_{6}+1\right) R_{3}+\left(R_{11}\left(k_{3}+1\right)+R_{13}\right) R_{10} y_{6}+R_{11}+\left(-k_{3}+1\right) R_{13}\right) . \\
& \begin{array}{c}
N\left(Z_{11}\right)=y_{5}\left[R_{3}\left(R_{11}+R_{13}\right) R_{12}\left(R_{10} y_{6}+1\right)\right]+\left(\left(R_{12}+R_{10}\right) y_{6}+1\right) R_{3}\left(R_{11}+R_{13}\right) \\
\quad d=y_{5}\left[k_{3} R_{12}\left(R_{10} R_{11} y_{6}-R_{13}\right)+\left(\left(R_{12}+R_{10}\right) y_{6}+1\right) R_{3}\left(R_{11}+R_{13}\right)+\right. \\
\left.\quad+R_{12}\left(R_{10} y_{6}+1\right)\left(R_{3}+R_{11}+R_{13}\right)\right]+\left(\left(R_{12}+R_{10}\right) y_{6}+1\right)\left(R_{11}\left(k_{3}+1\right)+R_{3}+R_{13}\right)
\end{array}
\end{aligned}
$$

Таким образом, функция искомой чувствительности $S_{y_{5}}^{K}$ включает выражение (16) при $Y=y_{5}$ и формулы (17)-(25), которые получены путем разложения 4-х схемных миноров: $d$ и $D(K)$ - определителей подсхемы 1 и схемы с эквивалентным генератором, $N\left(Z_{11}\right)$ и $N\left(\kappa_{1}\right)$ - числителей входного сопротивления и коэффициента передачи подсхемы 1. В то же время по обобщенной формуле Боде с бисекцией схемных определителей [9] требуется раскрыть 12 схемных миноров (строка 2 в таблице).

Автоматизация формирования символьных функций чувствительности может быть выполнена на основе программы символьного анализа и диагностики линейных электрических цепей CirSym, разработанной B.B. Филаретовым [12]. Online-сервис программы CirSym имеется на сайте http://intersyn.net/cirsym.html.

\section{ЗАКЛЮЧЕНИЕ}

1. Предложена безызбыточная формула для нахождения символьной функции относительной чувствительности линейных электрических цепей по частям на основе частных чувствительностей параметров ЭМГ.

2. Формула чувствительности на основе ЭМГ требует раскрытия многократно меньшего числа определителей подсхем (схемных миноров), чем обобщенная формула чувствительности Боде в сочетании с формулой бисекции схемных определителей, что позволяет существенно уменьшить вычислительные затраты при формировании функций чувствительности.

\section{СПИСОК ЛИТЕРАТУРЫ}

1. Боде Г. Теория цепей и проектирование усилителей с обратной связью : пер. с англ. - М. : ГИИЛ, 1948. $641 \mathrm{c}$.

2. Sensitivity analysis of analog circuits based on a modified nodal approach / L. Mandache, M. Iordache, L. Dumitriu, I. Sirbu, D. Niculae // International Conference on Optimization of Electrical and Electronic Equipment. 2014, Moieciu, Romania. May 22-24. pp. 83-88.
3. Asenova I.N. Calculation of first-, second-order and multiparameter symbolic sensitivity of active circuits by using nullor model and modified Coates flow graph // International Journal of microelectronics and computer science. 2011. Vol. 2, no. 4. pp. 129-135.

4. Balik F., Rodanski B. Calculation of symbolic sensitivities for large-scale circuits in the sequence of expressions form via the transimpedance method // Analog Integrated Circuits and Signal Processing. 2004. Vol. 40. pp. 265-276.

5. Тимкин Ю.В. Анализ электронных схем методом двунаправленных графов. - М. : Энергоатомиздат, 1985. -256 с.

6. Курганов С.А., Филаретов В.В. Обобщенные безызбыточные формулы для чувствительности схемных функций линейных электрических цепей // Электричество.- 2017. -№ 2.- С. 44-50.

7. Курганов С. А., Недорезов М. В., Филаретов В.В. Оптимизация разностной формулы чувствительности линейных электрических цепей // In memoriam: Василий Андреевич Андреев : сб. памяти Андреева В.А. Ульяновск : УлГТУ, 2018. - С. 211-220.

8. Шакиров М.А. Преобразования и диакоптика электрических цепей. - Л. : Изд-во Ленингр. ун-та, 1980. $196 \mathrm{c.}$.

9. Курганов С.А., Недорезов М.В., Филаретов В.В. Анализ чувствительности каскадных электронных схем делением их на части // Синтез, анализ и диагностика электронных цепей : междунар. сб. науч. тр. - Ульяновск : УлГТУ, 2018. - Вып. 15. - С. 64-76.

10. Курганов Д.С., Курганов С.А., Филаретов В.В. Символьный анализ линейных электрических цепей по частям на основе схемно-алгебраической редукции // Известия вузов. Электромеханика, 2011. - № 5. - С. 9-15.

11. Курганов С.А., Филаретов В.В. Неявный принцип наложения воздействий в линейных электрических цепях // Электричество. - 2005. - № 1. - С. 32-43.

12. Филаретов В.В. Программа символьного анализа CIRSYM: история создания, структура и функции // Синтез, анализ и диагностика электронных цепей : между- 
нар. сб. науч. тр. - Ульяновск : УлГТУ, 2012. - Вып. 10. C. 158-171.

\section{REFERENCES}

1. Bode G. Teoriia tsepei i proektirovanie usilitelei $s$ obratnoi sviaziu, per. s angl. [Network Analysis and Feedback Amplifier Design, transl. from Engl.]. Moscow, GIIL Publ., 1948. 641 p.

2. Mandache L., Iordache M., Dumitriu L., Sirbu I., Niculae D. Sensitivity Analysis of Analog Circuits Based on a Modified Nodal Approach. International Conference on Optimization of Electrical and Electronic Equipment, 2014, Moieciu, Romania, pp. 83-88.

3. Asenova I.N. Calculation of First-, Second-Order and Multiparameter Symbolic Sensitivity of Active Circuits by Using Nullor Model and Modified Coates Flow Graph. International Journal of Microelectronics and Computer Science, 2011, vol. 2, no. 4, pp. 129-135.

4. Balik F., Rodanski B. Calculation of Symbolic Sensitivities for Large-Scale Circuits in the Sequence of Expressions Form via the Transimpedance Method. Analog Integrated Circuits and Signal Processing, 2004, vol. 40, pp. 265-276.

5. Timkin lu.V. Analiz elektronnykh skhem metodom dvunapravlennykh grafov [Electronic Circuits Analysis using the Bidirectional Graphs Technique]. Moscow, Energoatomizdat Publ., 1985. 256 p.

6. Kurganov S.A., Filaretov V.V. Obobshchennye bezyzbytochnye formuly dlia chuvstvitelnosti skhemnykh funktsii lineinykh elektricheskikh tsepei [Generalized Breakeven Formula for the Sensivity of Circuit Functions of Linear Electric Circuits]. Elektrichestvo [Electrical Technology Russia]. 2017, no. 2, pp. 44-50.

7. Kurganov S. A., Nedorezov M. V., Filaretov V.V. Optimizatsiia raznostnoi formuly chuvstvitelnosti lineinykh elektricheskikh tsepei [Optimization of the Difference
Formula of the Linear Electric-Circuit Sensitivity]. In memoriam: Vasilii Andreevich Andreev. Sb. pamiati Andreeva V.A. [In memoriam of Vasilii Andreevich Andreev. Studies in Honor of Andreev V.A.], Ulyanovsk, UISTU Publ., 2018, pp. 211-220.

8. Shakirov M.A. Preobrazovaniia $i$ diakoptika elektricheskikh tsepei [Transformations and Diacoptics of Electric Circuits]. Leningrad, Leningr. Univers. Publ., 1980. $196 \mathrm{p}$.

9. Kurganov S.A., Nedorezov M.V., Filaretov V.V. Analiz chuvstvitelnosti kaskadnykh elektronnykh skhem deleniem ikh na chasti [Analysis of Cascade Electronic Circuits Sensitivity using Sectioning Technique]. Sintez, analiz $i$ diagnostika elektronnykh tsepei. Mezhdunar. sb. nauch. tr. [Synthesis, Analysis, and Diagnostics of Electronic Circuits. Int. Proc.]. Ulyanovsk, UISTU Publ., 2018, iss. 15, pp. 64-76.

10. Kurganov D.S., Kurganov S.A., Filaretov V.V. Simvolnyi analiz lineinykh elektricheskikh tsepei po chastiam na osnove skhemno-algebraicheskoi reduktsii [Symbolic Analysis in Parts of Linear Electric Circuits on the Basis of Circuit-Algebraic Reduction]. Izvestiia vuzov. Elektromekhanika [Russian Electromechanics. Sci. and Tech. Joutnal], 2011, no. 5, pp. 9-15.

11. Kurganov S.A., Filaretov V.V. Neiavnyi printsip nalozheniia vozdeistvii v lineinykh elektricheskikh tsepiakh [An Implicit Superposition Principle for Actions in Linear Electric Circuits]. Elektrichestvo [Electrical Technology Russia], 2005, no. 1, pp. 32-43.

12. Filaretov V.V. Programma simvolnogo analiza CIRSYM: istoriia sozdaniia, struktura i funktsii [CIRSYM Symbolic Analysis Program: Creation History, Structure and Functions]. Sintez, analiz $i$ diagnostika elektronnykh tsepey. Mezhdunar. sb. nauch. tr. [Synthesis, Analysis, and Diagnostics of Electronic Circuits. Int. Proc.]. Ulyanovsk, UISTU, 2012, iss. 10, pp. 158-171. 